\title{
Overcoming Practical Limitations to Probe Electronic Structure in Novel Quantum Materials
}

Berit Goodge ${ }^{1}$, Danfeng $\mathrm{Li}^{2}$, Kyuho Lee ${ }^{2}$, Motoki Osada ${ }^{2}$, Bai Yang Wang ${ }^{2}$, Harold Y. Hwang ${ }^{2}$ and Lena Kourkoutis $^{1}$

${ }^{1}$ Cornell University, Ithaca, New York, United States, ${ }^{2}$ SLAC National Accelerator Laboratory, Stanford University, Stanford, California, United States

Scanning transmission electron microscopy (STEM) and electron energy loss spectroscopy (EELS) can provide detailed structural and chemical characterization across a wide range of materials and length scales. Especially when atomic lattice-scale variations can enhance or quench exotic material properties, STEM-EELS is a powerful tool for probing the chemical or electronic structure of these materials at the atomic scale. The push for such robust, sensitive, and localized characterization is enabled by significant and ongoing improvements to hardware throughout the STEM-EELS instrument. As a case study to highlight several of these advances, we discuss key technical components in a series of experiments revealing the electronic structure of the recently discovered superconducting $\mathrm{Nd}_{(1-\mathrm{x})} \mathrm{Sr}_{(\mathrm{x})} \mathrm{NiO}_{2}$ thin films [1].

In recent years both sub- $\AA$ spatial resolution and sub-eV energy resolution have become nearly routine for many instruments thanks to higher-order aberration correction [2] and gun or monochromator improvements. Reliably interpretable data, however, requires not only sufficient resolution but also sufficient signal. In a real experiment, material considerations often limit the usable electron dose, posing new challenges for the practical application of these techniques. All EELS experiments presented here were conducted with a $\sim 1 \AA$ probe, a scan pixel size of $\sim 0.1 \AA^{2}$ and a dwell time of $0.5 \mu \mathrm{s} / \mathrm{px}$. For the sake of simplicity we therefore discuss the corresponding electron dose rate in terms of probe current. Measurements were made at $100 \mathrm{kV}$ in an aberration-corrected Nion UltraSTEM with a Gatan Quefina spectrometer. In the nickelate films, even a relatively modest current of $27 \mathrm{pA}$ causes significant modification to the O-K edge (Fig. 1a,b). In order to avoid these beam-induced effects, O-K edge data was therefore collected with beam currents of 9-13 pA. At such low current no changes were observed during sequential acquisitions, however, the signal-to-noise ratio (SNR) is severely limited even in minutes-long EELS acquisitions (Fig. 1c). Producing sufficiently high SNR spectra suitable for detailed analysis instead requires total summed acquisition times of up to several hours.

Alone, long acquisitions are not inherently difficult. In this case, however, they are significantly complicated by the simultaneous need for high spatial localization. HAADF STEM reveals some of the crystalline inhomogeneities within a nickelate film (Fig. 1d). Certain variations are clearly identifiable in standard imaging based on their crystalline structure, while others are not and can instead only be distinguished by their electronic signature (Fig. 1d,e). Furthermore, several different phases can be found within tens of nanometers in a single region. Sampling only the relevant phase thus requires sufficient spatial resolution and localization to both identify and avoid signals from other phases. For limited dose rates, this hinges on the ability to reliably scan a single few-nm region for up to 1 hour without significant spatial drift. Compared to side-entry designs, an in-column sample stage can offer a more than twofold reduction of sample drift [3].

With sufficiently high SNR spectra, it is possible to probe the electronic structure of a system through its energy loss near edge structure (ELNES). In some cases, such as distinguishing between different crystalline phases in Fig. 1a, ELNES changes may be quite obvious. In many cases, however, differences 
are much more subtle, either in edge shape or energy shift, requiring careful alignment and calibration of the energy axes across multiple experiments. For core-loss data — when the zero-loss peak (ZLP) is not recorded - the absolute energy of a spectrum is often calibrated from nearby known edges. In these cases, using very high spectrometer dispersions to maximize energy resolution can result in an undesirable tradeoff decreasing the total simultaneously collectable energy range.

Figure 2 shows spectra collected on a FEI Titan Themis monochromated X-FEG at $120 \mathrm{kV}$ with a Gatan $\mathrm{K} 2$ direct electron detector (DED) operated in electron counting mode [4-6]. The energy spread of the probe was measured to be $0.19 \mathrm{eV}$ as the zero-loss peak (ZLP) full-width at half-maximum (FWHM) over vacuum with a spectrometer dispersion of $0.01 \mathrm{eV} / \mathrm{ch}$. Using a dispersion of $0.025 \mathrm{eV} / \mathrm{ch}$ on the DED results in an effective energy resolution of $0.21 \mathrm{eV}$ with a total energy range of $\sim 93 \mathrm{eV}$. Because the point spread function (PSF) of the DED helps reduce energy broadening in the detector, a dispersion of $0.1 \mathrm{eV}$ increases the simultaneously accessible energy range by a factor of 4 while preserving an effective energy resolution of $0.29 \mathrm{eV}$ ZLP FWHM (Fig. 2a). With this energy resolution and range, the core-loss NiL2,3 spectra in Fig. 2b are aligned by the simultaneously acquired Nd-M4,5 edge at $978 \mathrm{eV}$ to reveal a subtle shift to higher energies in the infinite-layer $\mathrm{NdNiO}_{2}$. Particularly for experiments with time or dose limitations, the simultaneous collection of several edges can facilitate dynamic EELS experiments, tracking correlated changes across multiple components through space or time.

Optimization of experimental conditions and hardware for high spatial and spectral resolution STEMEELS was necessary to reveal the electronic signature of superconducting nickelate thin films. Far from unique, this case demonstrates the practical importance of marrying spatial resolution, energy resolution, and overall system stability not just within a single instrument, but simultaneously within a single experiment. Continued progress across hardware components - gun, optics, stage, spectrometer, and detector - will drive further advances in STEM-EELS and its application across all fields of both biological and physical sciences. [7] 

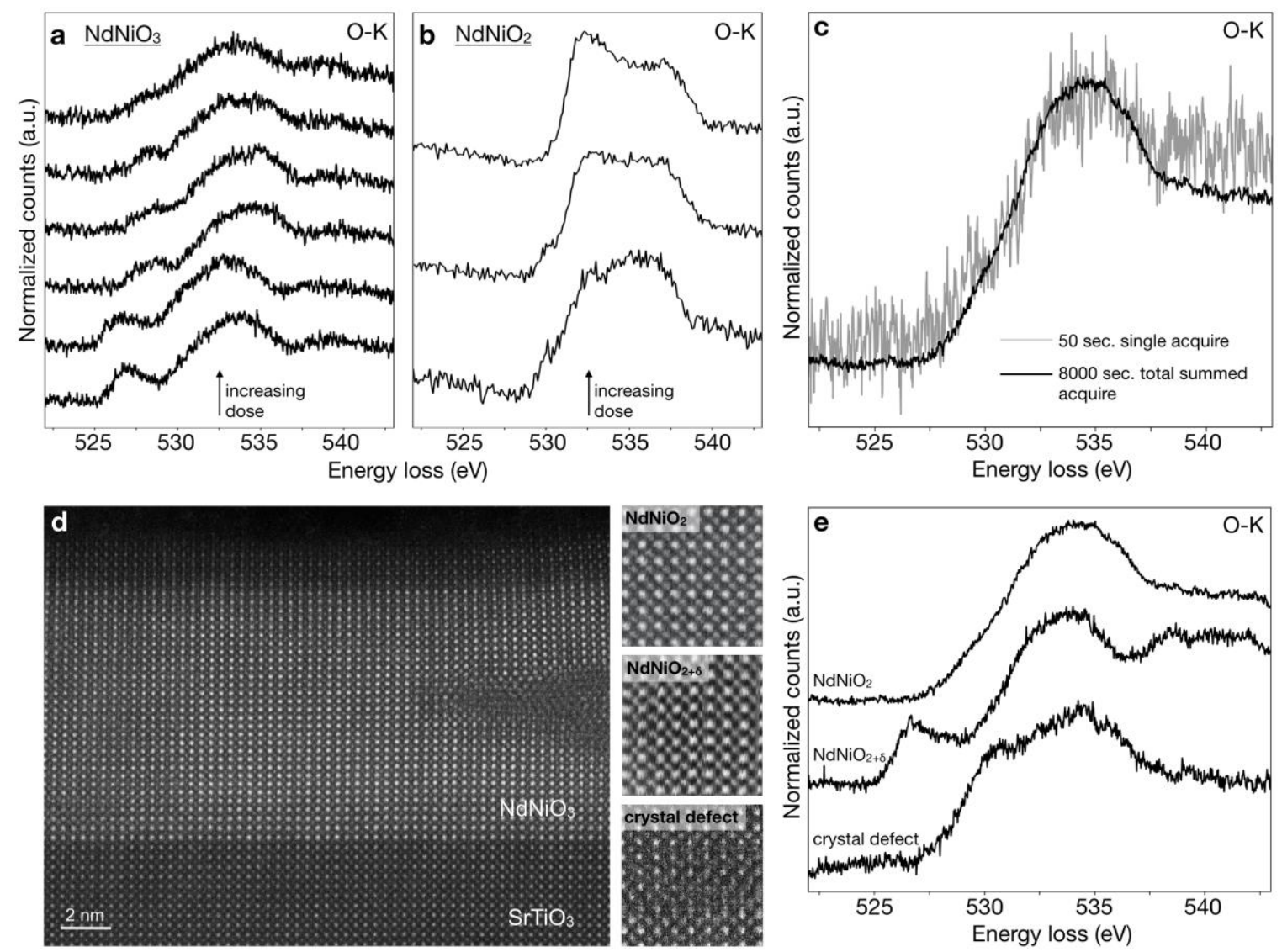

Figure 1. Recent EELS experiments on superconducting $\mathrm{Nd}(1-\mathrm{x}) \mathrm{SrxNiO} 2$ thin films demonstrate the need to combine high spatial and energy resolution with overall system stability in the STEM. a Using a probe current of $27 \mathrm{pA}$ at $100 \mathrm{kV}$, beam-induced modification to the $\mathrm{O}-\mathrm{K}$ edge of $\mathrm{NdNiO} 3$ is observed in a series of consecutive $200 \mathrm{sec}$. acquisitions of the same few-nm region. b Similar damage effects are also observed in NdNiO2. c Decreasing the probe current by more than half to $10 \mathrm{pA}$ prevents any observed beam damage, but requires much longer total acquisition times to build sufficient SNR for careful analysis. $\mathrm{d}$ Inhomogeneities in the thin film sample necessitate highly localized measurements at the few-nm scale. Some regions can be easily identified by HAADF STEM imaging, such as the crystalline defect shown on the right, while others are crystallographically indistinguishable, such as $\mathrm{NdNiO} 2$ and $\mathrm{NdNiO} 2+\delta$. e Distinct ELNES fingerprints in the O-K edge of the three phases shown in d. Often, all three phases can co-exist within several $\mathrm{nm}$, necessitating highly localized stability across extended acquisitions. 

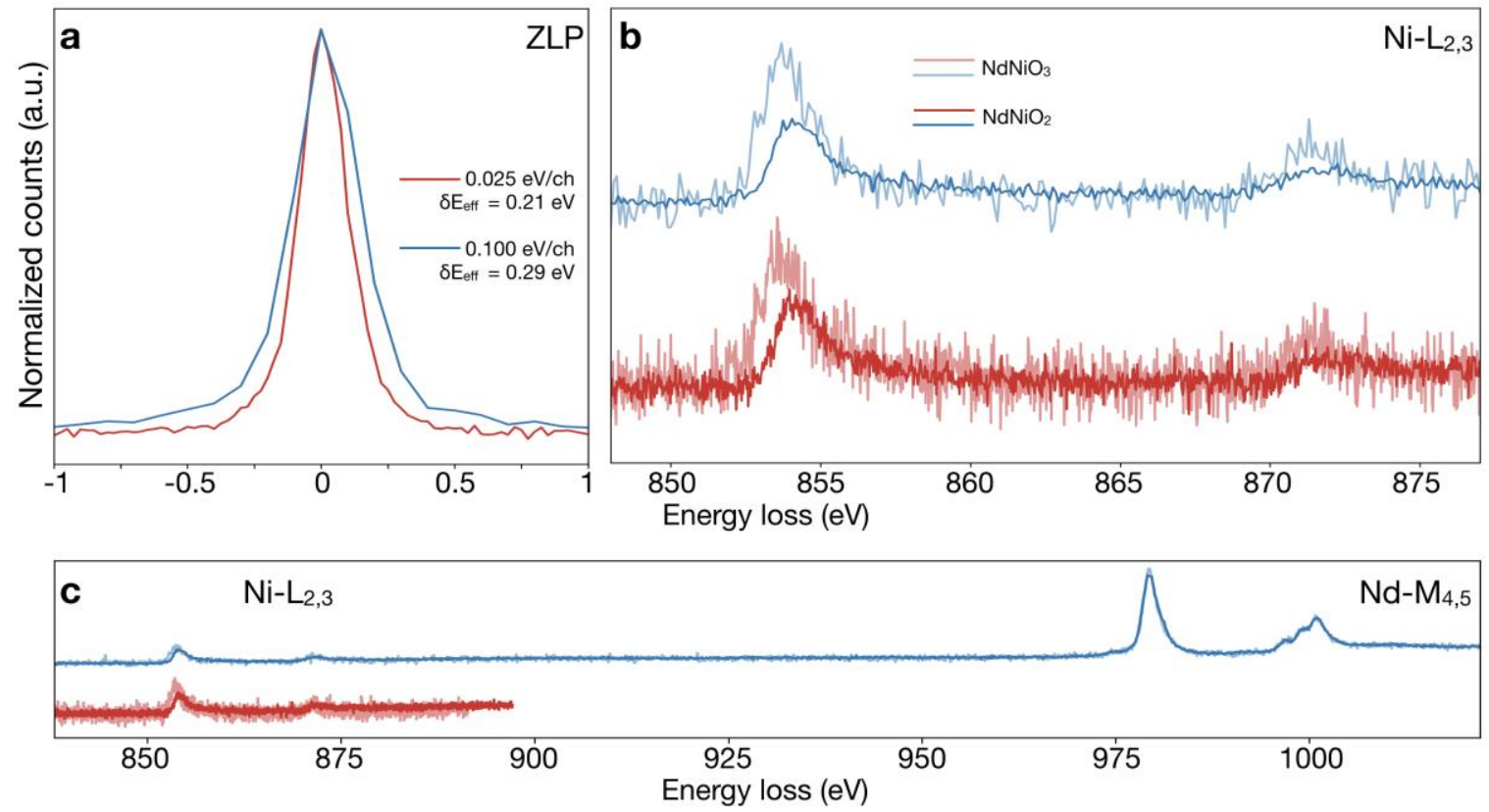

Figure 2. Both energy resolution and range are important for core-loss EELS experiments. a Representative ZLPs from a $120 \mathrm{kV}$ monochromated X-FEG measured on a DED with spectrometer dispersions of 0.025 (red) and 0.1 (blue) $\mathrm{eV} / \mathrm{ch}$, achieving effective energy resolutions $\delta$ Eeff of 0.21 and $0.29 \mathrm{eV}$, respectively. $\mathrm{b}$ Both dispersions are sufficient to resolve a subtle shift and broadening in the NiL3 edge from the perovskite $\mathrm{NdNiO} 3$ (light) to the infinite layer $\mathrm{Nd}(1-\mathrm{x}) \mathrm{SrxNiO} 2$ phase (dark). c Using a $0.1 \mathrm{eV} / \mathrm{ch}$ dispersion increases the total energy range from less than 100 to over $370 \mathrm{eV}$, so that the $\mathrm{Nd}-$ M4,5 edge can be used for absolute energy calibration.

\section{References}

[1] Li, et al. Nature 572, (2019), p. 624-627.

[2] Muller, et al. Science 319, (2008), p. 1073-1075.

[3] Goodge, et al. arXiv:2001.11581.

[4] Hart, et al. Scientific Reports 7, (2017), p. 8243.

[5] Goodge, et al. Microsc. Microanal. 24 S1, (2018), p. 1844-1845.

[6] Cheng, et al. Microsc. Microanal. 25 S2, (2019), p. 586-587.

[7] Supported by DOD AFOSR (FA 9550-16-1-0305), NSF (DMR-1539918, DMR-1429155, DMR1719875), DOE (DE-AC02-76SF00515), and the Gordon \& Betty Moore Foundation (GBMF4415). 